\title{
Alimentação de peixes na microbacia do Ribeirão Grande, Serra da Mantiqueira oriental, SP
}

\author{
Francisco Manoel de Souza Braga ${ }^{1,2}$ \& Leandro Muller Gomiero ${ }^{I}$ \\ ${ }^{1}$ Departamento de Zoologia, Instituto de Biociências, Universidade Estadual Paulista - UNESP, \\ Av. 24-A, 1515, CP 199, CEP 13506-900, Rio Claro, SP, Brasil, www.rc.unesp.br \\ ${ }^{2}$ Autor para correspondência: Francisco Manoel de Souza Braga, e-mail: fmsbraga@rc.unesp.br
}

BRAGA, F.M.S. \& GOMIERO, L.M. Feeding of fishes in the Ribeirão Grande system, eastern serra da Mantiqueira, SP. Biota Neotrop., 9(3): http://www.biotaneotropica.org.br/v9n3/en/ abstract?article+bn04009032009.

\begin{abstract}
Feeding habits of fishes were studied in the Ribeirão Grande system (22 $47^{\prime} 08^{\prime \prime} \mathrm{S}$ and $\left.45^{\circ} 28^{\prime} 17^{\prime \prime} \mathrm{W}\right)$, state of São Paulo. Stomach content of each specimen was analyzed through the grade of feeding preference, and classified as autochthonous and alochthonous, according to their origin. The eleven species of fishes were classified into one of three trophic categories: herbivore, when species fed almost entirely on periphyton; Pareiorhina rudolphi was the only species assigned to this category. Omnivores consumed plant and animal material; this category included Astyanax scabripinnis and Gymnotus pantherinus. Benthic insectivores, when species fed predominantly on immature forms of benthic insects; included in this category were Characidium lauroi, C. alipioi, Trichomycterus itatiayae, T. immaculatus, T. iheringi, Neoplecostomus microps, Taunaya bifasciata and Imparfinis minutus. Insects were the main food item consumed by the species in the Ribeirão Grande system, occurring predominance of autochthonous prey in relation to alochthonous prey.

Keywords: feeding of fish, alochthonous, autochthonous, atlantic forest.
\end{abstract}

BRAGA, F.M.S. \& GOMIERO, L.M. Alimentação de peixes na microbacia do Ribeirão Grande, serra da Mantiqueira oriental, SP. Biota Neotrop., 9(3): http://www.biotaneotropica.org.br/v9n3/pt/ abstract?article+bn04009032009.

Resumo: Foi estudado o hábito alimentar dos peixes da microbacia do Ribeirão Grande, estado de São Paulo ( $22^{\circ} 47^{\prime} 08^{\prime \prime} \mathrm{S}$ e $45^{\circ} 28^{\prime} 17^{\prime \prime} \mathrm{O}$ ). O conteúdo estomacal foi analisado usando-se o grau de preferência alimentar, e classificado como autóctone e alóctone, de acordo com a sua origem. As espécies de peixes foram classificadas em três categorias tróficas: herbívoras, espécies que comeram principalmente perifiton; Pareiorhina rudolphi foi a única espécie classificada nesta categoria. Onívoras, espécies que consumiram itens de origem animal e vegetal; esta categoria incluiu Astyanax scabripinnis e Gymnotus pantherinus. Insetívoras bentônicas, espécies que consumiram predominantemente formas imaturas bentônicas de insetos; foram incluídas nesta categoria Characidium lauroi, C. alipioi, Trichomycterus itatiayae, T. immaculatus, T. iheringi, Neoplecostomus microps, Taunaya bifasciata e Imparfinis minutus. Os insetos foram os principais itens alimentares consumidos pelas espécies de peixes na microbacia do Ribeirão Grande, ocorrendo predominância de presas autóctones em relação às presas alóctones.

Palavras-chave: alimentação de peixes, alóctone, autóctone, mata atlântica. 


\section{Introdução}

Os corpos de águas continentais apresentam uma grande diversidade de forma, de estrutura física e de composição química, variando desde riachos com menos de um metro de largura, por alguns centímetros de profundidade, até rios com alguns milhares de quilômetros de extensão e algumas dezenas de metros de profundidade; pequenas lagoas ou brejos rasos, lagos de dolinas profundos que se formam em regiões calcárias ou lagos formados por falhas geológicas, mais profundos ainda, como os grandes lagos africanos. Um corpo de água continental em movimento é conhecido como lótico, quando a correnteza é rápida, ou lêntico, quando a correnteza é lenta, o que os limnólogos europeus definem como rithron e potamon, respectivamente (Wootton 1992).

Em qualquer uma dessas circunstâncias, existe uma íntima relação entre o corpo de água e o terreno por onde ele flui (Allan 1995). Em áreas montanhosas de terreno cristalino, o trajeto do riacho é orientado por diáclases do terreno, tornando o fluxo torrentoso e inconstante, enquanto que em áreas de rochas sedimentares moles, geralmente em planícies, o rio traça o seu trajeto de forma meandrante, sendo as margens inundáveis denominadas de várzeas (Braga 2004).

Nestes sistemas hídricos, o ecossistema é doador e receptor de nutrientes alóctones, sem os quais a produção primária e cadeias alimentares não poderiam ser sustentadas (Jeffries 2000). Quando a vegetação ciliar ou a morfologia do canal são modificados, a biota aquática passa a ser afetada pelo transporte elevado de sedimento, aumento da temperatura, quebra da cadeia trófica e decréscimo na diversidade de hábitats (Karr \& Schlosser 1978).

Em riachos de cabeceira o fluxo rápido de água e a baixa disponibilidade de luz inibem o desenvolvimento da vegetação enraizada e o desenvolvimento abundante de fitoplâncton, e a produção primária é feita por algas e musgos incrustados no leito (Wootton 1992). Com o aumento da ordem do riacho aumenta a oferta de itens autóctones, enquanto que em trechos de cabeceira a oferta de alimento é principalmente de origem alóctone (Lotrich 1973, Bishop 1973 apud Wootton 1992). Além disso, em rios oligotróficos o material terrestre carreado para a água constitui um importante recurso alimentar para os peixes (Pusey \& Artington 2003, Melo et al. 2004).

A cobertura florestal tem um efeito importante sobre a ecologia alimentar de peixes de riachos. Em riachos onde as margens são sombreadas, a principal fonte de energia vem de vegetais e de invertebrados terrestres (Wootton 1992). Embora a contribuição de itens autóctones aumente com a ordem dos riachos, a principal fonte de energia vem de vegetais de origem alóctone (Bishop op. cit.). Nestes ambientes a contribuição de material alóctone é muito importante para a ictiofauna, disponibilizando tanto direta como indiretamente os itens alimentares (Rezende \& Mazzoni 2005).

Invertebrados que se alimentam de matéria orgânica grosseiramente particulada (CPOM), proveniente da vegetação ripária, são mais abundantes em trechos de cabeceira (Schlosser 1982). Com o aumento do volume do riacho, os coletores que utilizam-se da matéria orgânica mais fina (FPOM) passam a predominar (Vannote et al. 1980, Allan 1995). Nas tramas alimentares existentes em riachos tropicais envolvendo peixes e macroinvertebrados, Uieda \& Motta (2007) destacam a importância no sistema e a participação do detrito e alga como fonte de alimento para organismos que irão ser consumidos pelos peixes, como larvas e ninfas de insetos aquáticos.

Em um sistema lótico de riacho de montanha, que por suas características físicas tende a ser limitado espacialmente (raso e estreito) e inconstante, formando poções e rápidos, a fonte de energia na cadeia é principalmente de origem alóctone (Uieda \& Kikuchi 1995, Rezende \& Mazzoni 2003, Dufech et al. 2003, Graciolli et al. 2003, Rezende \& Mazzoni 2006a,b, Pinto \& Uieda 2007).
A microbacia do Ribeirão Grande é formada por riachos que descem as encostas da serra da Mantiqueira oriental, no município de Pindamonhangaba (SP) que vão desaguar na margem esquerda do rio Paraíba do Sul. Nos últimos anos foram realizados vários estudos nessa microbacia, objetivando-se conhecer a distribuição de peixes (Braga \& Andrade 2005), adaptações desenvolvidas pelas espécies (Braga 2004), biologia e estrutura populacional de Characidium spp. (Braga 2005a,b, Braga 2006, Braga et al. 2007), aspectos da reprodução e alimentação de Neoplecostomus microps (Steindachner 1877) (Braga et al. 2008) e de Pareiorhina rudolphi (Miranda Ribeiro 1911) (Braga et al. 2009).

Neste trabalho analisamos a alimentação de peixes, e a contribuição do alimento de origem alóctone e autóctone, em uma comunidade de peixes de riacho na microbacia do Ribeirão Grande, serra da Mantiqueira oriental, uma das poucas regiões do estado de São Paulo onde as condições da água ainda são boas.

\section{Material e Métodos}

A área de estudo situa-se nas encostas da serra da Mantiqueira oriental, entre os municípios de Campos do Jordão e Pindamonhangaba, estado de São Paulo (22 47'08' S e 45 $28^{\circ}$ ' 17'” O). Os riachos que formam a microbacia do Ribeirão Grande descem as encostas da serra da Mantiqueira num gradiente altitudinal que vai de $1.800 \mathrm{~m}$ até a planície do Vale do Paraíba, com altitude ao redor de $550 \mathrm{~m}$ (Braga \& Andrade 2005). Os riachos descem a escarpa ainda recoberta em vários trechos pela mata pluvial tropical (Hueck 1972), entremeada por campos abertos, principalmente na região do pediplano e planície do Vale do Paraíba (Braga 2004). O elevado grau de inclinação da escarpa, a decomposição do gnaisse rico em biotita, a elevada pluviosidade que ocorre nos meses de verão e a pequena profundidade do regolito que se apoia sobre a rocha ígnea, tudo isso associado com áreas desmatadas, tem provocado o deslizamento do regolito, expondo a rocha nua (tálus). Esse deslizamento pode alterar o percurso de riachos (Braga 2004), e tem sido verificado com frequiência na área de estudo. A interação entre as margens e áreas de entorno com o leito do riacho são importantes para a geração do fluxo de energia utilizada pelas diferentes categorias de organismos aquáticos (Jeffries 2000), e mudanças que possam ocorrer neste sentido irão produzir alterações nas comunidades aquáticas (Karr \& Schlosser 1978).

Foram realizadas coletas trimestrais, sendo duas em 2001 (julho e outubro) e duas em 2002 (janeiro e abril), utilizando-se um aparelho de pesca elétrica acoplado a um gerador com capacidade de 300 a $500 \mathrm{~V}$ e 8,7 A, com corrente direta. Foram amostrados cinco riachos que formam a microbacia do Ribeirão Grande, desde a encosta até a planície do Vale do Paraíba. Em cada riacho percorria-se um trecho de $50 \mathrm{~m}$, com uma repetição. A descrição pormenorizada dos riachos amostrados e suas características encontram-se em Braga \& Andrade (2005).

Os exemplares capturados foram fixados em formol a $10 \%$, acondicionados em sacos plásticos e em laboratório, após alguns dias, transferidos para álcool a 70\%. Os indivíduos foram identificados e agrupados de acordo com a espécie. Os estômagos em grau de repleção total foram selecionados para análise de seu conteúdo. Os itens alimentares foram identificados, sob microscópio estereoscópio, a partir de guias de identificação e livro texto sobre organismos aquáticos (Bicudo \& Bicudo 1970, Borror \& DeLong 1969, Needham \& Needham 1982).

Para a análise dos itens alimentares identificados foi utilizado o método do grau de preferência alimentar (GPA), descrito em Braga (1999). Para isso foram inicialmente atribuídos quatro valores numéricos aos itens alimentares identificados. O valor 4 é atribuído quando em um estômago ocorrer apenas um item alimentar. Quando ocorrer 
mais de um item por estômago, adota-se o seguinte procedimento: o item que preponderar receberá o valor 3 , o que ocorrer com baixa abundância, o valor 1 e o que ocorrer em abundância intermediária, o valor 2; depois desta etapa, determina-se então o grau de preferência alimentar (GPA) que é dado por $\mathrm{Si} / \mathrm{N}$, onde $\mathrm{Si}$ é a soma dos valores atribuídos à abundância do item alimentar (i) nos estômagos e $\mathrm{N}$ o número total de estômagos analisados. Os valores estimados para o grau de preferência alimentar (GPA) referente a cada item alimentar tem os seguintes significados:

GPA = 4: o item em questão tem preferência absoluta;

$3 \leq \mathrm{GPA}<4$ : o item em questão tem alto grau de preferência;

$2 \leq \mathrm{GPA}<3$ : o item em questão é preferencial, mas outros itens também são ingeridos;

$1 \leq \mathrm{GPA}<2$ : o item em questão é secundário;

$0<\mathrm{GPA}<1$ : o item em questão é ocasional.

Este método atribui valores à quantidade de cada item ingerido por estômago e o classifica em categorias de preferência. Nesta análise foram consideradas as espécies que apresentaram mais de seis estômagos repletos de alimento.

\section{Resultados e Discussão}

Na microbacia do Ribeirão Grande foram descritas 37 espécies de peixes (Braga \& Andrade 2005), das quais 11 foram estudadas no presente trabalho, por apresentarem estômagos em número considerado suficiente para as análises, ou seja, um mínimo de seis estômagos com grau de repleção total. Das dez espécies mais abundantes na microbacia do Ribeirão Grande (Braga \& Andrade 2005), oito foram estudadas neste trabalho, estando as outras três entre as 19 mais abundantes (Tabela 1). Todas, com exceção de Pareiorhina rudolphi e de Neoplecostomus microps, alimentaram-se de itens alóctones e autóctones, mas houve preferência por itens de origem autóctone (Figura 1). Dos itens autóctones, houve preferência alimentar por larvas de Diptera (Chironomidae e Simuliidae) e ninfas de Plecoptera. Larvas de Diptera ocorreram com valores elevados para o grau de preferência alimentar em quase todas as espécies analisadas, enquanto que ninfas de Plecoptera, embora não ocorrendo como itens de relevância na preferência alimentar, ocorreram em dez das onze espécies estudadas (Tabela 1 e Figura 1). Segundo Bispo et al. (2002), Plecoptera é um bioindicador de águas de boa qualidade, ocorrendo em regiões de altitudes elevadas, com densa mata ribeirinha e com pouca ação antrópica.

A única espécie que teve preferência por itens alóctones foi Astyanax scabripinnis (Jenyns 1842), que ingeriu insetos terrestres; Gymnotus pantherinus (Steindachner 1908) ingeriu vegetais superiores, sem ser possível fazer a distinção entre vegetal superior terrestre ou aquático.

As espécies foram grupadas em três categorias tróficas, conforme seus graus de preferências alimentares: herbívoros (Pareiorhina rudolphi), onívoros (Astyanax scabripinnis e Gymnotus pantherinus), insetívoros bentônicos (Characidium lauroi Travassos 1949, C. alipioi Travassos 1955, Trichomycterus itatiayae Miranda Ribeiro 1906, T. immaculatus (Eigenmann \& Eigenmann 1889), T. iheringi (Eigenmann 1917), Neoplecostomus microps, Taunaya bifasciata (Eigenmann \& Norris 1900) e Imparfinis minutus (Lütken 1874)).

A predominância de espécies generalistas e oportunistas nos ambientes fluviais tropicais reflete as oscilações espaciais e temporais na abundância relativa dos itens alimentares (Abelha et al. 2001). A plasticidade trófica das espécies oportunistas pode ser causada pela necessidade de se utilizar uma variedade de itens alimentares em condições adversas, sendo este um fator limitante para a sobrevivências dessas espécies nesses ambientes (Teixeira et al. 2005).

Pinto \& Uieda (2007) destacam a importância de itens alóctones na organização trófica em comunidades de peixes de riachos tropicais. Porém, os itens alóctones nem sempre passam a entrar imediatamente na cadeia trófica de peixes; muitos itens, em especial os de origem vegetal, passam por etapas sucessivas de transformações para então serem ingeridos indiretamente pelos peixes (Uieda \& Motta 2007). Webster \& Benfield (1986), que estudaram os processos pelos quais passa um item alóctone (planta vascular), demonstram em detalhes essas etapas, que inicia-se com a lixivia do folhiço transportado do chão da floresta para o riacho, seguido pela colonização microbiana e posterior fragmentação mecânica pela ação de invertebrados. Nesta etapa, é intensa a ação de larvas e ninfas de insetos que vão processar

Tabela 1. Valores do grau de preferência alimentar de cada item ingerido por peixes da microbacia do Ribeirão Grande.

Table 1. Values of the degree of food preference of each item ingested by fish of the microbacia of Ribeirão Grande.

\begin{tabular}{|c|c|c|c|c|c|c|c|c|c|c|c|}
\hline Itens Alimentares & 1 & 2 & 3 & 4 & 5 & 6 & 7 & 8 & 9 & 10 & 11 \\
\hline Insetos terrestres & 0,4 & 0,1 & - & - & - & 2,1 & 0,3 & - & 0,4 & 1,0 & 0,5 \\
\hline Frutos & - & - & - & - & - & 0,4 & - & - & - & - & - \\
\hline Vegetal superior & - & 0,2 & 0,2 & - & - & 0,1 & 0,1 & 0,4 & - & 0,7 & 2,9 \\
\hline Perifiton & - & - & - & 4,0 & - & - & - & - & - & - & - \\
\hline Oligochaeta & - & - & - & - & - & - & 0,1 & 0,7 & - & 0,7 & - \\
\hline Ostracoda & 0,1 & - & - & - & - & - & - & - & - & 0,2 & - \\
\hline Decapoda & - & - & - & - & - & - & - & - & - & 1,0 & - \\
\hline Teleostei & - & - & - & - & - & - & - & - & - & - & 0,2 \\
\hline Insetos aquáticos & 0,1 & 0,1 & - & - & - & 0,1 & 0,4 & - & 0,6 & - & 0,3 \\
\hline Ninfas de Odonata & 0,1 & 0,4 & - & - & - & - & 0,2 & - & 0,1 & - & - \\
\hline Ninfas de Plecoptera & 0,4 & 0,5 & 1,2 & 0,2 & 0,8 & 0,2 & 0,1 & 0,4 & 0,9 & 1,0 & - \\
\hline Ninfas de Ephemeropetra & 1,5 & 0,2 & 2,1 & - & - & 0,1 & 0,4 & 0,7 & - & - & - \\
\hline Larvas de Megaloptera & - & 0,1 & - & - & - & 0,1 & - & 0,5 & - & - & - \\
\hline Larvas de Trichoptera & 0,5 & 1,3 & 0,2 & - & - & 0,3 & 1,2 & 0,4 & 0,3 & - & - \\
\hline Larvas de Diptera & 1,9 & 1,9 & 2,0 & - & 2,6 & - & 0,9 & - & 2,3 & 1,0 & - \\
\hline
\end{tabular}

1 - Characidium lauroi; 2 - Characidium alipioi; 3 - Trichomycterus itatiayae; 4 - Pareiorhina rudolphi; 5 - Neoplecostomus microps; 6 Astyanax scabripinnis; 7 - Imparfinis minutus; 8 - Taunayia bifasciata; 9 - Trichomycterus immaculatus; 10 - Trichomycterus iheringi; and 11 - Gymnotus pantherinus. 


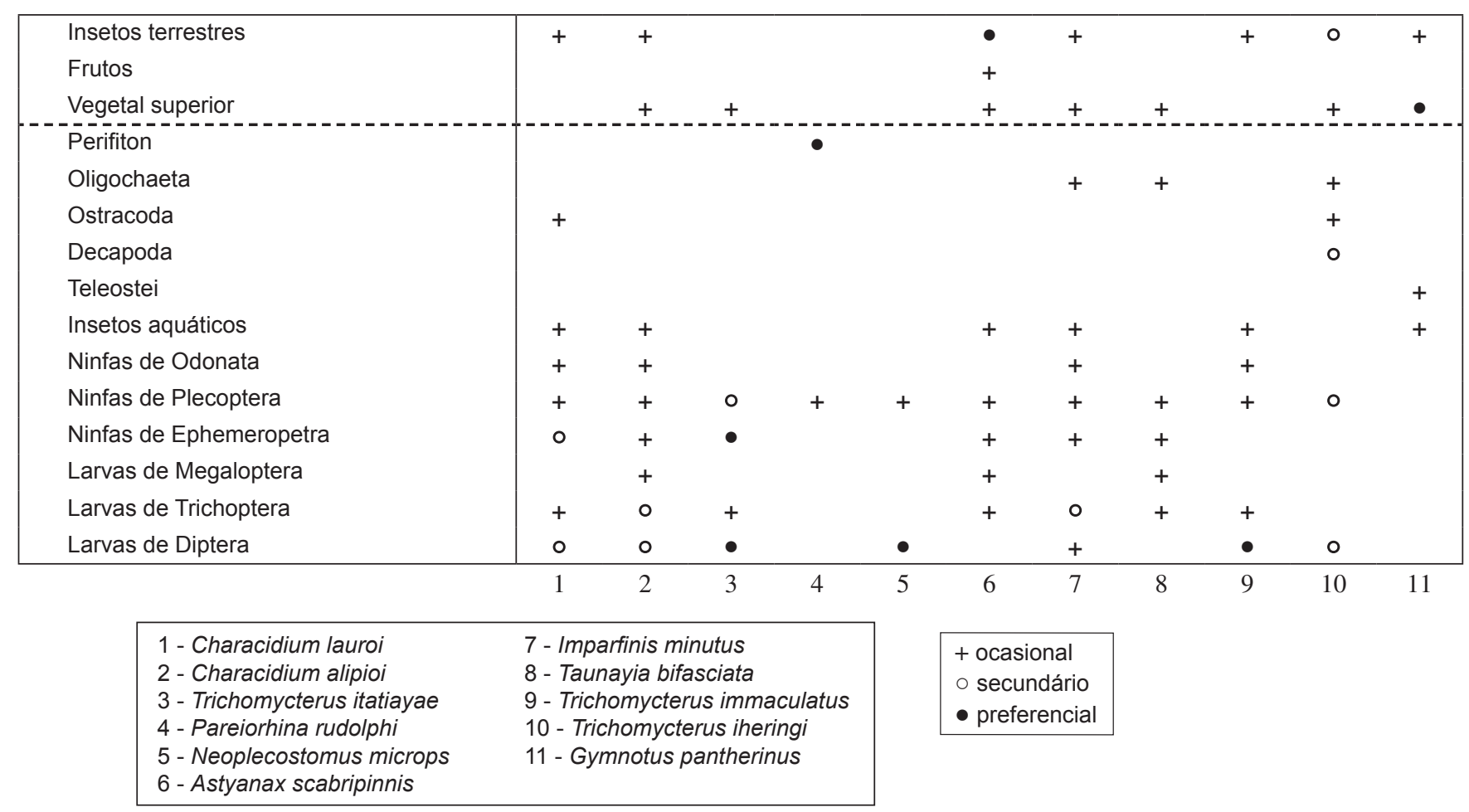

Figura 1. Espécies abundantes na microbacia do Ribeirão Grande e suas dietas, arranjadas seguindo o grau de preferência alimentar. A linha horizontal pontilhada separa os itens de origem alóctone (acima) e autóctones (abaixo).

Figure 1. Abundant species in the microbacia of Ribeirão Grande and their diets, arranged following the degree of food preference. The stippled horizontal line separates the items of origin alochthonous (above) and autochthonous (below).

a fonte alimentar de diversas e sucessivas maneiras, como mastigar e retalhar (larvas de Trichoptera, ninfas de Plecoptera), coletar partículas em suspensão (larvas de Chironomidae e de Simuliidae), pastar sobre o material vegetal particulado que serve de substrato para o perifiton (ninfas de Ephemeroptera, larvas de Trichoptera) e exercer a ação predadora sobre outros grupos (ninfa de Odonata, larva de Megaloptera), e assim estabelecer uma longa e diversificada cadeia envolvendo invertebrados consumidores em riachos (Cummins 1973, Wallace \& Merrit 1980, Allan 1995). A importância de itens alóctones fica então demonstrada pela participação de organismos autóctones na cadeia trófica, como larvas e ninfas de insetos aquáticos, que por sua vez serão itens importantes na alimentação dos peixes de riachos na microbacia do Ribeirão Grande.

Os peixes não escolhem locais de alimentação ao acaso, e podem influenciar significativamente comunidades de invertebrados bentônicos (Gillian et al. 1989). Mesmo possuindo uma plasticidade trófica ampla (Nikolsky 1963, Lowe-McConnell 1975, Wootton 1992, Gerking 1994), o hábito onívoro não pode ser generalizado para muitas espécies de peixes, devido não só ao comportamento e fisiologia, como também à estrutura do aparato bucal. Portanto, as condições impostas por esses fatores prevalecem sobre o oportunismo em muitas espécies de peixes.

Várias espécies de Characidae utilizam-se de recursos alimentares de matas ciliares (Dufech et al. 2003, Graciolli et al. 2003, Rezende \& Mazzoni 2003). Rezende \& Mazzoni (2006b) não encontraram diferenças no consumo de itens de origem alóctone e autóctone na alimentação de Bryconamericus microcephalus (Miranda Ribeiro 1908), em um riacho sombreado de mata Atlântica, mas encontraram predominância de presas autóctones em ambientes abertos. O posi- cionamento da boca terminal e a diversidade de padrões dentários multicuspidados apresentado por espécies da sub-família Tetragonopterinae possibilitam a elas uma grande amplitude do espectro alimentar, explorando itens de diversas origens, tanto alóctones como autóctones (Costa \& Braga 1993). Em outros rios com sombreamento da Mata Atlântica foi constatada a importância dos itens alóctones e autóctones na alimentação de Brycon opalinus (Cuvier 1819) Characidae (Gomiero et al. 2008), Oligosarcus hepsetus (Cuvier 1829) - Characidae (Botelho et al. 2007) e Rhamdia quelen (Quoy \& Gaimard 1824) - Heptapteridae (Gomiero et al. 2007).

Por outro lado, espécies menos generalistas mantêm suas dietas alimentares baseadas nas mesmas categorias de itens, independentemente do local que habitam. Em riachos da sub-bacia do rio Corumbataí, Characidium zebra Eigenmann 1909 consumiu apenas itens autóctones (Gomiero \& Braga 2008). Na microbacia do Ribeirão Grande, Characidium lauroi distribui-se da encosta ao pediplano, em áreas mais recobertas por mata ciliar, e C. alipioi distribui-se do pediplano à planície do vale, em áreas mais abertas (Braga 2005a). No entanto, a alimentação dessas espécies constitui-se de invertebrados bentônicos, tanto em áreas de encosta-pediplano, como nas do pediplano-vale (Braga 2005b). Prevalece neste caso a adaptação que essas espécies têm em obter o alimento de origem autóctone, sem levar em conta o alimento que possa vir de outra fonte.

Os ambientes de riachos de montanha, embora sejam oligotróficos, limitados pela produção primária abundante, e também espacialmente pela pequena área que ocupam, alocam muitas espécies que utilizam-se principalmente de fontes alimentares de origem autóctone. Braga et al. (2008) e Braga et al. (2009) estudaram a alimentação de Neoplecostomus microps e de Pareiorhina rudolphi, e constataram 
que mesmo sendo simpátricas alimentam-se de fontes autóctones distintas: $N$. microps tem uma dieta feita principalmente de larvas de Diptera, e $P$. rudolphi de perifiton (Figura 1), sendo que a morfologia bucal é fundamental para a escolha dos itens alimentares.

\section{Conclusões}

Muitas das espécies que habitam riachos de montanha na microbacia do Ribeirão Grande, serra da Mantiqueira oriental, fazem uso de itens alimentares autóctones, como larvas e ninfas de insetos principalmente, consumindo em menor escala itens alóctones. A contribuição de itens alóctones se faz de maneira indireta, servindo como fonte de energia para organismos heterótrofos como larvas e ninfas de insetos, que servirão de alimento aos peixes.

\section{Agradecimentos}

Somos agradecidos à FAPESP e ao CNPq pelo suporte financeiro a este projeto e à Cemasi e Votorantim Celulose e Papel/VCP - Florestal, pelo apoio oferecido durante as atividades de campo na fazenda São Sebastião do Ribeirão Grande.

Referências Bibliográficas

\section{Referências Bibliográficas}

ABELHA, M.C.F., AGOSTINHO, A.A. \& GOULART, E. 2001. Plasticidade trófica em peixes de água doce. Acta Sci. 23(2):425-434.

ALLAN, J.D. 1995. Stream ecology: structure and function of running waters. Chapman and Hall, London.

BICUDO, C.E.M. \& BICUDO, R.M.T. 1970. Algas de águas continentais brasileiras (chave ilustrada para identificação de gêneros). Funbec, São Paulo.

BISPO, P.C., FROEHLICH, C.G. \& OLIVEIRA, L.G. 2002. Spatial distribution of plecoptera nymphs in streams of a mountainous area of Central Brazil. Rev. Bras. Biol. 62(3):409-417.

BORROR, D.J. \& DeLONG, D.M. 1969. Introdução ao estudo dos insetos. Editora Edgar Blucher, São Paulo.

BOTELHO, M.L.L.A., GOMIERO, L.M. \& BRAGA, F.M.S. 2007. Feeding of Oligosarcus hepsetus (Cuvier, 1829) (Characiformes) in the Serra do Mar State Park - Santa Virgínia Unit, São Paulo, Brazil. Rev. Bras. Biol. 67(4):741-748.

BRAGA, F.M.S. 1999. O grau de preferência alimentar: um método qualitativo e quantitativo para o estudo do conteúdo estomacal de peixes. Acta Sci. 21(2):291-295.

BRAGA, F.M.S. 2004. Hábitat, distribuição e aspectos adaptativos de peixes da microbacia do Ribeirão Grande, estado de São Paulo, Brasil. Acta Sci. 26(1):31-36.

BRAGA, F.M.S. 2005a. Spatial distribution of characidiin fishes (Teleostei, Crenuchidae), in the Ribeirão Grande system, a tributary of Paraíba do Sul river basin, Brazil. Acta Sci. 27(3):259-263.

BRAGA, F.M.S. 2005b. Feeding and condition factor of characidiin fish in Ribeirão Grande system, southeastern Brazil. Acta Sci. 27(3):271-276.

BRAGA, F.M.S. 2006. Aspectos da reprodução no gênero Characidium Reinhardt, 1867 (Crenuchidae, Characidiinae), na microbacia do Ribeirão Grande, serra da Mantiqueira, sudeste do Brasil. Acta Sci. 27(4):259-263.

BRAGA, F.M.S. \& ANDRADE, P.M. 2005. Distribuição de peixes na microbacia do Ribeirão Grande, serra da Mantiqueira oriental, São Paulo, Brasil. Iheringia, Sér. Zool. 95(2):121-126.

BRAGA, F.M.S., GOMIERO, L.M. \& SOUZA, U.P. 2008. Aspectos da reprodução e alimentação de Neoplecostomus microps, (Loricariidae, Neoplecostominae) na microbacia do Ribeirão Grande, serra da Mantiqueira oriental (SP). Acta Sci. 30(4): 455-463.

BRAGA, F.M.S., GOMIERO, L.M. \& SOUZA, U.P. 2009. Biologia populacional de Pareiorhina rudolphi (Loricariidae, Hypostominae) na microbacia do Ribeirão Grande, serra da Mantiqueira oriental, estado de São Paulo. Acta Sci. 31(1):79-88.

COSTA, F.E.S. \& BRAGA, F.M.S. 1993. Estudo da alimentação natural de Astyanax bimaculatus, A. schubarti e Moenkhausia intermedia (Characidae, Tetragonopterinae) na represa de Barra Bonita, rio Piracicaba (SP). Rev. Unimar. 15(2):117-134.

CUMMINS, K.W. 1973. Trophic relations of aquatic insects. Annu. Rev. Entomol. 18:183-206.

DUFECH, A.P.F., AZEVEDO, M.A. \& FIALHO, C.B. 2003. Comparative dietary analysis of two populations of Mimagoniates rhocharis (Characidae, Glandulocaudinae) from two streams of southern Brazil. Neotrop. Ichthyol. 1(1): 67-74.

GERKING, S.D. 1994. Feeding ecology of fish. Academic Press, San Diego.

GILLIAN, J.F., FRASER, D.F. \& SABAT, A.M. 1989. Strong effects of foraging minnows on a stream benthic invertebrate community. Ecology. 70(2):445-452.

GOMIERO, L.M. \& BRAGA, F.M.S. 2008. Feeding habits of the ichthyofauna in a protected area in the state of São Paulo, southeastern Brazil. Biota Neotrop. 8(1):1-8.

GOMIERO, L.M., SOUZA, U.P. \& BRAGA, F.M.S. 2007. Reprodução e alimentação de Rhamdia quelen (Quoy \& Gaimard, 1824) em rios do Núcleo Santa Virgínia, Parque Estadual da Serra do Mar, São Paulo, SP. Biota Neotrop. 7(3):1-7.

GOMIERO, L.M., MANZATTO, A.G. \& BRAGA, F.M.S. 2008. The role of riverine forests for food supply for the omnivorous fish Brycon opalinus Cuvier, 1819 (Characidae) in the Serra do Mar, southeast Brazil. Rev. Bras. Biol. 68(2):321-328.

GRACIOLLI, G.M., AZEVEDO, M.A. \& MELO, F.A.G. 2003. Comparative study of the Glandulocaudinae and Tetragonopterinae (Ostariophysi: Characidae) in a small stream in southern Brazil. Stud. Neotr. Fauna Environ. 38(2):95-110.

HUECK, K. 1972. As florestas da América do Sul. Editora da Universidade de Brasília; Editora Polígono S.A., São Paulo.

JEFFRIES, R.L. 2000. Allocthonous inputs: integrating populations changes and food-web dynamics. Tree. 15(1):19-22.

KARR, J.R. \& SCHLOSSER, I.J. 1978. Water resources and the land water interface. Science. 201(4352):229-234.

LOTRICH, V.A. 1973. Growth, production, and community composition of fishes inhabiting a first-, second-, and third-order stream of eastern Kentucky. Ecol. Monogr. 43(3):377-397.

LOWE-McCONNELL, R.H. 1975. Fish communities in tropical freshwaters. Longman, New York.

MELO, C.E., MACHADO, F.A. \& PINTO-SILVA, V. 2004. Feeding habits of fish from a stream in the savanna of Central Brazil, Araguaia basin. Neotrop. Ichthyol. 2(1):37-44.

NEEDHAM, J.G. \& NEEDHAM, P.R. 1982. Guia para el estudio de los seres vivos de las aguas dulces. Editora Reverté S.A., Barcelona.

NIKOLSKY, G.V. 1963. The ecology of fishes. Academic Press, London.

PINTO, T.L.F. \& UIEDA, V.S. 2007. Aquatic insects selected as food for fishes of a tropical stream: are there spacial and seasonal differences in their selectivity? Acta Limnol. Bras. 19(1):67-78.

PUSEY, B.J. \& ARTHINGTON, A.H. 2003. Importance of the riparian zone to the conservation and management of freshwater fish: a review. Mar. Freshw. Res. 54(1):1-16.

REZENDE, C.F. \& MAZZONI, R. 2003. Aspectos da alimentação de Bryconamericus microcephalus (Characidae, Tetragonopterinae) no córrego Andorinha, Ilha Grande-RJ. Biota Neotrop. 3(1):1-6.

REZENDE, C.F. \& MAZZONI, R. 2005. Seasonal variation in the input of allochthonous matter in an Atlantic rain forest stream, Ilha Grande-RJ. Acta Limnol. Bras. 17(2):167-175.

REZENDE, C.F. \& MAZZONI, R. 2006a. Disponibilidade e uso de recursos alóctones por Bryconamericus microcephalus (Miranda Ribeiro) 
(Actinopterygii, Characcidae), no córrego Andorinha, Ilha Grande, Rio de Janeiro, Brasil. Rev. Bras. Zool. 23(1): 218-222.

REZENDE, C.F. \& MAZZONI, R. 2006b. Contribuição da matéria autóctone e alóctone para a dieta de Bryconamericus macrocephalus (Miranda Ribeiro) (Actinopterygii, Characidae), em dois trechos de um riacho de mata atlântica, Rio de Janeiro, Brasil. Rev. Bras. Zool. 23(1):58-63.

SCHLOSSER, I.J. 1982. Fish community structure and function along two habitat gradients in a headwater stream. Ecol. Monogr. 52(4):395-414.

TEIXEIRA, T.P., PINTO, B.C.T., TERRA, B.F., ESTILIANO, E.O., GRACIA, D. \& ARAÚJO, F.G. 2005. Diversidade das assembléias de peixes nas quatro unidades geográficas do rio Paraíba do Sul. Iheringia, Sér. Zool. 95(4):347-357.

UIEDA, V.S. \& KIKUCHI, R.M. 1995. Entrada de material alóctone (detritos, vegetais e invertebrados terrestres) num pequeno curso de água corrente na cuesta de Botucatu, São Paulo. Acta Limnol. Bras. 7:105-114.
UIEDA, V.S. \& MOTTA, R.L. 2007. Trophic organization and food web structure of southeastern Brazilian streams: a review. Acta Limnol. Bras. 19(1):15-30.

VANNOTE, R.L., MINSHALL, G.W., CUMMINS, K.W., SEDELL, J.R. \& CUSHING, C.E. 1980. The river continuum concept. Can. J. Fish. Aquat. Sci. 37(1):130-137.

WALLACE, J.B. \& MERRIT, R.W. 1980. Filter-feeding ecology of aquatic insects. Annu. Rev. Entomol. 25:103-132.

WEBSTER, J.R. \& BENFIELD, E.F. 1986. Vascular plant breakdown in freshwater ecosystem. Annu. Rev. Ecol. System. 17:567-594.

WOOTTON, R.J. 1992. Fish ecology. Chapman and Hall, New York.
Recebido em 18/04/09 Versão reformulada em 17/08/09 Publicado em 29/09/09 